\title{
SURFACE AND SUBSURFACE RESIDUAL STRESSES AFTER MACHINING AND THEIR ANALYSIS BY X-RAY DIFFRACTION
}

\begin{abstract}
Process specifications and working procedures widely used by the aerospace and automotive industries require surface analysis by machining and specify the process parameters such as type, destructive measuring or simulations. Destructive measuring or simulation carried out in order to optimise and later to verify the process parameters are a very indirect way of measurement. While they are performed on simulation only similar in composition and elastic properties to that of the actual part to be machined, they almost never match all the important conditions of the process such as the shape of the real part or the residual stress prior to the treatment. Consequently the residual stresses and their depth distribution after the machining may differ very significantly from those required by the technologist. The only reliable way to verify that the operation has produced the desired effect is to actually measure the stresses in the machined component.
\end{abstract}

Keywords: Surface, residual stresses, X-ray diffraction, machining.

\section{Introduction}

Residual stresses are an integral part of manufactured workpieces, whether they are introduced deliberately, as a part of the design, as a by-product of a process carried out during the manufacturing process, or are present as the product of the component's service history. Residual stresses are additive with the stresses existing in the workpieces as a result of service loads. Clearly, they may be considered beneficial to the workpieces and therefore desirable, they may be irrelevant and can be ignored, or they are a potential detriment to the workpieces and their continued service life. Given an adequate history, the magnitude of residual stresses in parts that are in service can be considered as indicators of the workpiece's deterioration [1].

Obviously, to realize the benefits of understanding the residual stresses in parts and structures, tools are needed to measure them. Several techniques are available, with varying degrees of sophistication. Some of them are rather limited in their application, but one stands out as having widespread applications and being readily available.

X-ray diffraction is applicable to crystalline materials, which include virtually all metals and their alloys, and most ceramic materials. It is a non-destructive detection technology in many applications, and is widely accepted by the engineering community, being the subject of SAE and ASTM publications, which provide reliable sources of information on methods to ensure repeatability and reliability in the results of measurements. Because individual mea- surements are non-destructive, they can be replicated to demonstrate their statistical reliability.

This article will look closely at the methodology of residual stress measurement by X-ray diffraction, explore the characteristics of measurements performed using modern X-ray methods, and offer a few practical examples [2].

\section{Principles of X-Ray Diffraction Stress Measurement}

Macroscopic stresses, which extend over distances that are large relative to the grain size of the material, are of general interest in design and failure analysis. Macroscopic stresses are tensor quantities, with magnitudes varying with direction at a single point in a component. The macroscopic stress for a given location and direction is determined by measuring the strain in that direction at a single point. When macroscopic stresses are determined in at least three known directions, and a condition of plane stress is assumed, the three stresses can be combined using Mohr's circle for stress to determine the maximum and minimum residual stresses, the maximum shear stress, and their orientation relative to a reference direction. Macroscopic stresses strain many crystals periodically in the surface. This periodical distortion of the crystal lattice shifts the angular position of the diffraction peak selected for residual stress measurement [1].

Microscopic stresses are scalar properties of the sample, such as percent of cold work or hardness, which are without direction

\footnotetext{
* Andrej Czan, Eva Tillova, Jan Semcer, Jozef Pilc

Faculty of Mechanical Engineering, University of Zilina, Slovakia, E-mail: andrej.czan@fstroj.uniza.sk
} 
and result from imperfections in the crystal lattice. Microscopic stresses are associated with strains within the crystal lattice that traverse distances on the order or less than the dimensions of the crystals. Microscopic stresses change within the crystal lattice, altering the lattice spacing and broadening the diffraction peak. Macroscopic stresses and microscopic stresses can be determined individually from the diffraction peak position and breadth [1].

Figure 1 describes the diffraction of a monochromatic beam of $X$-rays at a high diffraction angle $2 \theta$ from the surface of a stressed sample for two orientations of the sample relative to the X-ray beam. The angle $\psi$, defining the orientation of the sample surface, is the angle between the normal of the surface and the incident and diffracted beam bisector, which is also the angle between the normal to the diffracting lattice planes and the sample surface [3].

Diffraction occurs at an angle $2 \theta$, which is defined by Bragg's Law: $n \lambda=2 d \sin \theta$, where $\mathrm{n}$ is a number denoting the order of diffraction, $\lambda$ is the $\mathrm{X}$-ray wavelength, $d$ is dimension of the lattice spacing of crystal planes, and $\theta$ is the diffraction angle. For the monochromatic X-rays produced by the metallic target of an X-ray tube, the wavelength is known to 1 part in 105 . Any change in the lattice spacing $\mathrm{d}$ results in a corresponding shift in the diffraction angle $2 \theta$. Figure 1 a shows the sample in the $\psi=0$ orientation. The presence of a tensile stress in the sample results in a Poisson's ratio contraction, reducing the lattice spacing and slightly increasing the diffraction angle, $2 \theta$. If the sample is then rotated through some known angle $\psi$ (Fig. 1b), the tensile stress present in the surface increases the lattice spacing over the stress-free state and decreases $2 \theta$. Measuring the change in the angular position of the diffraction peak for at least two orientations of the sample defined by the angle $\psi$ enables calculation of the stress present in the sample surface lying in the plane of diffraction, which contains the incident and diffracted X-ray beams. To measure the stress in different directions at the same point, the sample is rotated about its surface normal so that the direction of interest coincides with the diffraction plane. Because only the elastic strain changes the mean lattice spacing, only elastic strains are measured using X-ray diffraction for the determination of macroscopic stresses. When the elastic limit is exceeded, further strain results in dislocation motion, disruption of the crystal lattice, and the formation of microscopic stresses, but no additional increase in macroscopic stress. Although residual stresses result from non-uniform plastic deformation, all residual macrostresses remaining after deformation are necessarily elastic.

The residual stress determined using X-ray diffraction is the arithmetic average stress in a volume of material defined by the irradiated area, which may vary from square centimetres to square millimetres, and the depth of penetration of the X-ray beam. The linear absorption coefficient of the material for the radiation used governs the depth of penetration, which can vary considerably. However, in iron, nickel, and aluminium-based alloys, $50 \%$ of the radiation is diffracted from a layer approximately $0.005 \mathrm{~mm}$ deep for the radiations generally used for stress measurement. This shallow depth of penetration allows determination of macro and microscopic residual stresses as functions of depth, with depth resolution approximately 10 to 100 times that possible using other methods. Although in principle virtually any interplanar spacing may be used to measure strain in the crystal lattice, the availability of the wavelengths produced by commercial X-ray tubes limits the choice to a few possible planes. The choice of a diffraction peak selected for residual stress measurement impacts significantly on the precision of the method. The higher the diffraction angle, the greater the precision. Practical techniques generally require diffraction angles, $2 \theta$, greater than $120^{\circ}[4]$.

Plane-stress elastic model X-ray diffraction stress measurement is confined to the surface of the sample. Electropolishing is used

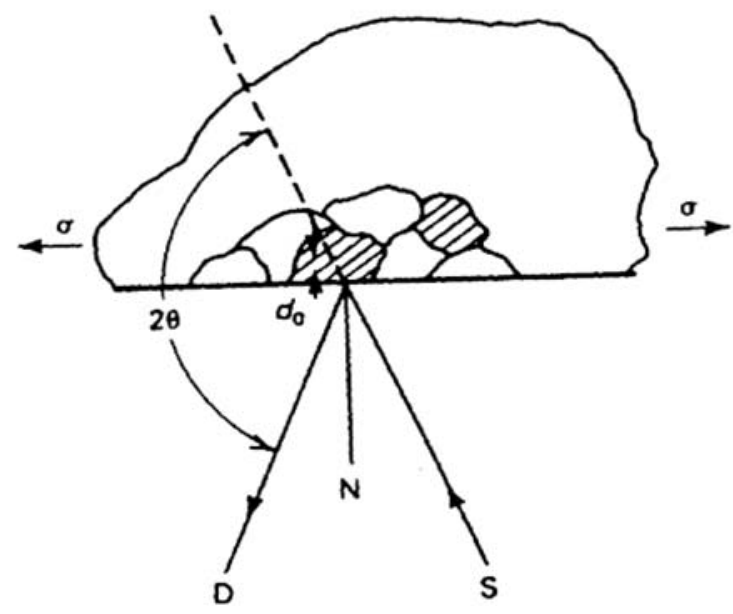

a)

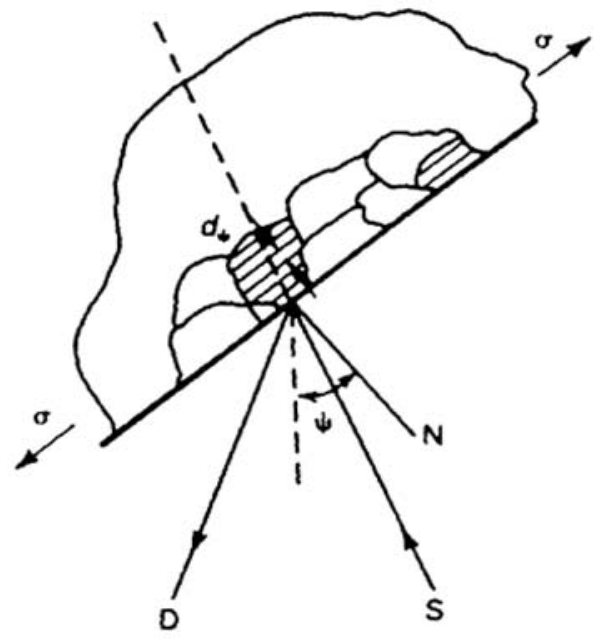

b)

(a) out rotation $\psi=0$. (b) angle of rotation $\psi=\psi$ sample rotated through some known angle $\psi, D$ - detector: $S$ - source. $N$ - normal to the surface.

Fig. 1 Principles of $X$-ray diffraction stress measurement [3] 
to expose new surfaces for subsurface measurement. In the exposed surface layer, a condition of plane stress is assumed to exist. That is, a stress distribution described by principal stresses $\sigma_{1}$ and $\sigma_{2}$ exists in the plane of the surface, and no stress is assumed perpendicular to the surface, $\sigma_{3}=0$. However, a strain component perpendicular to the surface $\varepsilon_{3}$ exists as a result of the Poisson's ratio contractions caused by the two principal stresses (Fig. 2) [5].

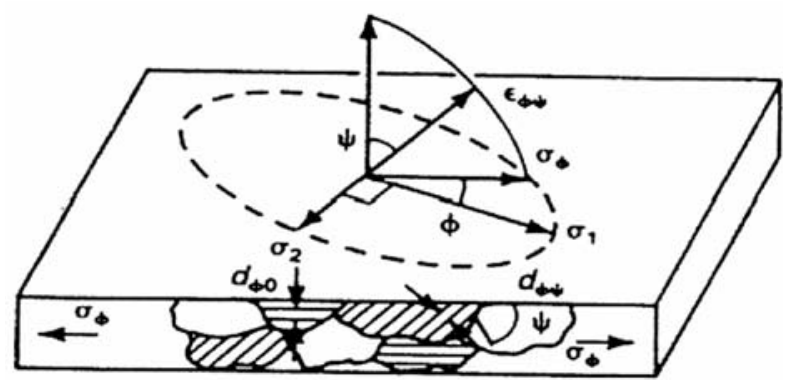

Fig. 2 Plane-stress elastic model [3]

The strain, $\varepsilon_{\varphi \psi}$ in the direction defined by the angles $\varphi$ and $\psi$ is:

$$
\varepsilon_{\varphi \psi}=\left[\frac{1+v}{E}\left(\sigma_{1} \alpha_{1}^{2}+\sigma_{2} \alpha_{2}^{2}\right)\right]-\left[\frac{v}{E}\left(\sigma_{1}+\sigma_{2}\right)\right]
$$

where $E$ is the modulus of elasticity, $v$ is the Poisson's ratio, and $\alpha_{1}$ and $\alpha_{2}$ are the angle cosines of the strain vector:

$$
\begin{aligned}
& \alpha_{1}=\cos \varphi \sin \psi \\
& \alpha_{2}=\sin \varphi \sin \psi
\end{aligned}
$$

Substituting for the angle cosines in Eq. 1 and simplifying enables the strain to be expressed in terms of the orientation angles:

$$
\begin{aligned}
& \varepsilon_{\varphi \psi_{\phi \psi}}=\left[\frac{1+v}{E}\left(\sigma_{1} \cos ^{2} \varphi+\sigma_{2} \sin ^{2} \varphi\right) \sin ^{2} \psi\right]- \\
& -\left[\frac{v}{E}\left(\sigma_{1}+\sigma_{2}\right)\right]
\end{aligned}
$$

If the angle $\psi$ is taken to be $90^{\circ}$, the strain vector lies in the plane of the surface, and the surface stress component, $\sigma_{\varphi}$ is:

$$
\sigma_{\varphi}=\left(\sigma_{1} \cos ^{2} \varphi\right)+\left(\sigma_{2} \sin ^{2} \varphi\right)
$$

Substituting Eq. 4 into Eq. 3 yields the strain in the sample surface at an angle $\varphi$ from the principal stress $\sigma_{1}$ :

$$
\varepsilon_{\varphi \psi}=\left[\frac{1+v}{E} \sigma_{\varphi} \sin ^{2} \psi\right]-\left[\left(\frac{v}{E}\right)\left(\sigma_{1}+\sigma_{2}\right)\right]
$$

Equation 5 relates the surface stress $\sigma_{\varphi}$, in any direction defined by the angle $\psi$, to the strain, $\in$, in the direction $(\varphi, \psi)$ and the principal stresses in the surface. If $d_{\varphi \psi}$ is the spacing between the lattice planes measured in the direction defined by $\varphi$ and $\psi$, the strain can be expressed in terms of changes in the linear dimensions of the crystal lattice: where $d_{0}$ is the stress-free lattice spacing. Substitution into Eq. 5 yields:

$$
\begin{aligned}
& \frac{d_{\varphi \psi}-d_{0}}{d_{0}}=\left[\left(\frac{1+v}{E}\right)_{(h k l)} \sigma_{\varphi} \sin ^{2} \psi\right]- \\
& -\left[\left(\frac{v}{E}\right)_{(h k l)}\left(\sigma_{1}+\sigma_{2}\right)\right]
\end{aligned}
$$

where the elastic constants $(1+v / E)_{(h k l)}$ and $(v / E)_{(h k l)}$ are not the bulk values but the values for the crystallographic direction normal to the lattice planes in which the strain is measured as specified by the Miller indices $(h k l)$. Because of elastic anisotropy, the elastic constants in the $(h k l)$ direction commonly vary significantly from the bulk mechanical values, which are an average over all possible directions in the crystal lattice. The lattice spacing for any orientation, then, is:

$$
\begin{aligned}
& d_{\varphi \psi}=\left[\left(\frac{1+v}{E}\right)_{(h k l)} \sigma_{\varphi} d_{0} \sin ^{2} \psi\right]- \\
& -\left[\left(\frac{v}{E}\right)_{(h k l)} d_{0}\left(\sigma_{1}+\sigma_{2}\right)+d_{0}\right]
\end{aligned}
$$

Equation 7 describes the fundamental relationship between lattice spacing and the biaxial stresses in the surface of the sample. The lattice spacing $d_{\varphi \psi}$, is a linear function of $\sin ^{2} \psi$.

The intercept of the plot at $\sin ^{2} \psi=0$ is:

$$
\begin{aligned}
& d_{\varphi 0}=d_{0}-\left(\frac{v}{E}\right)_{(h k l)} d_{0}\left(\sigma_{1}+\sigma_{2}\right)= \\
& =d_{0}\left[1-\left(\frac{v}{E}\right)_{(h k l)}\left(\sigma_{1}+\sigma_{2}\right)\right]
\end{aligned}
$$

which equals the unstressed lattice spacing, $d_{0}$, minus the Poisson's ratio contraction caused by the sum of the principal stresses. The slope of the plot is:

$$
\frac{\partial d_{\varphi \psi}}{\partial \sin ^{2} \psi}=\left(\frac{1+v}{E}\right)_{(h k l)} \sigma_{\varphi} d_{0}
$$

which can be solved for the stress $\sigma_{\phi}$ :

$$
\sigma_{\varphi}=\left(\frac{E}{1+v}\right)_{(h k l)} \frac{1}{d_{0}}\left(\frac{\partial d_{\varphi \psi}}{\partial \sin ^{2} \psi}\right)
$$

The X-ray elastic constants can be determined empirically, but the unstressed lattice spacing, $d_{0}$, is generally unknown. However, because $E$ » $\left(\sigma_{1}+\sigma_{2}\right)$, the value of $d_{\varphi 0}$ from Eq. 8 differs from $d 0$ by not more than $\pm 1 \%$, and $\sigma \phi$ may be approximated to this accuracy using:

$$
\sigma_{\varphi}=\left(\frac{E}{1+v}\right)_{(h k l)}\left(\frac{s_{1}-s_{2}}{2 R}\right)\left(\frac{\cot \theta}{\sin ^{2} \psi_{1}-\sin ^{2} \psi_{2}}\right)
$$

The method then becomes a differential technique, and no stress-free reference standards are required to determine $d_{0}$ for the biaxial stress case. The three most common methods of X-ray diffraction residual stress measurement, the single-angle, two-angle, and $\sin ^{2} \psi$ techniques, assume plane stress at the sample surface and are based on the fundamental relationship between lattice spacing and stress given in Eq. 7 [6].

The single-angle technique, or single-exposure technique, derives its name from early photographic methods that require a single 
exposure of the film. The method is generally considered less sensitive than the two-angle or $\sin ^{2} \psi$ techniques primarily because the possible range of $\psi$ is limited by the diffraction angle $2 \theta$ [7].

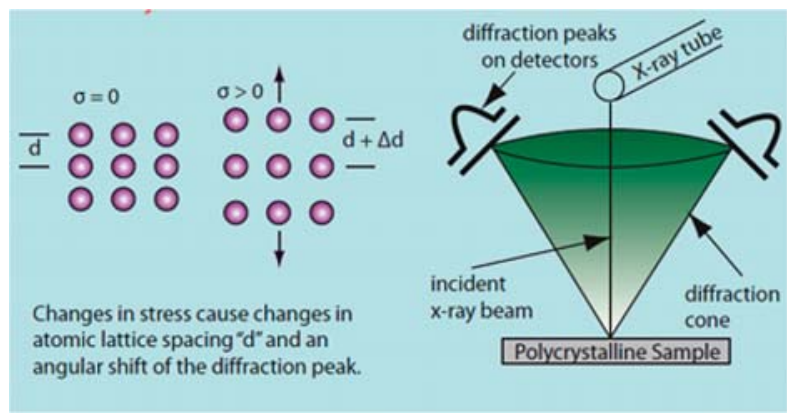

Fig. 3 Basic geometry of the single-angle technique for X-ray diffraction residual stress measurement [4]

Figure 3 shows the basic geometry of the method. A collimated beam of X-rays is inclined at a known angle, $\beta$, from the sample surface normal. X-rays diffract from the sample, forming a cone of diffracted radiation originating at point 0 . The diffracted X-rays are recorded using film or position-sensitive detectors placed on either side of the incident beam. The presence of a stress in the sample surface varies the lattice spacing slightly between the diffracting crystals shown at points 1 and 2 in Fig. 3, resulting in slightly different diffraction angles on either side of the X-ray beam. If detector $S 1$ and detector $S 2$ are the arc lengths along the surface of the film or detectors at a radius $\mathrm{R}$ from the sample surface, the stress is:

$$
\sigma_{\varphi}=\left(\frac{E}{1+v}\right)_{(h k l)}\left(\frac{s_{1}-s_{2}}{2 R}\right)\left(\frac{\cot \theta}{\sin ^{2} \psi_{1}-\sin ^{2} \psi_{2}}\right)
$$

The angles $\psi_{1}$, and $\psi_{2}$ are related to the Bragg diffraction angles $\theta_{1}, \theta_{2}$, and the angle of inclination of the instrument, $\beta$, by:

$$
\psi_{1}=\beta+\theta_{1}-\frac{\pi}{2} \text { and } \psi_{2}=\beta+\theta_{2}-\frac{\pi}{2}
$$

The precision of the method is limited by the principle that increasing the diffraction angle $2 \theta$ to achieve precision in the determination of lattice spacing reduces the possible range of $\sin ^{2} \psi$, lessening sensitivity. The single-angle technique is generally not used, except for film and position-sensitive detector apparatuses designed for high-speed measurement [7].

\section{Quantitative and Qualitative Stress Analysis}

The conventional way of measuring the surface residual stresses is X-ray diffraction (XRD). This is a well-established quantitative, absolute method and provides accurate stress values. In machined components the beneficial maximum compressive stresses are beneath the surface and thus to verify the result of the machining, evaluation below the surface is necessary. The measurement depth of the XRD method is limited to a few microns. To measure the subsurface stress by XRD requires successive electrochemical removal of material and repeated XRD measurements. Such a procedure is acceptable for laboratory evaluations on selected samples. Difficult to reach areas such as holes, fillets or roots of gears form an additional difficulty. The XRD is nevertheless irreplaceable in obtaining the true and complete picture of the effects of machining. Particularly, very steep stress gradients after machining of very hard steels are well resolved by this technique. It ought to be mentioned here that, in addition to the stress profile, also the effects of plastic deformation, work hardening or softening of the machined surface can be illustrated and quantified by the XRD measurement (Fig. 4).

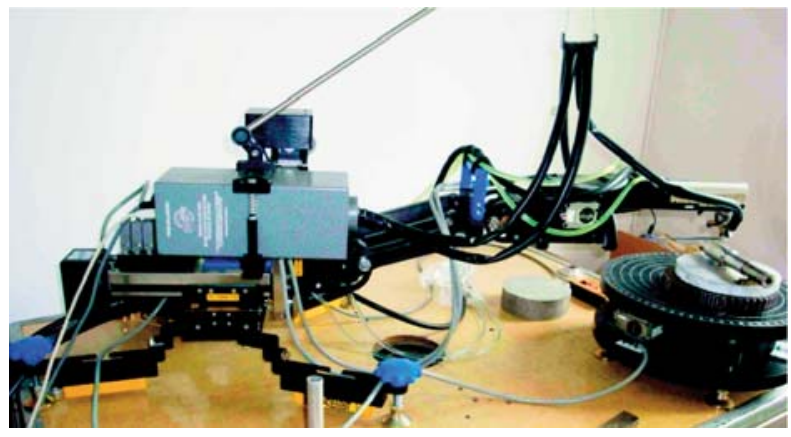

Fig. 4 Measurement system for residual stresses in X-ray diffraction

\section{Material and experimental technique}

Experimental studies were made on a high carbon rolled steel bar (60 mm diameter and $500 \mathrm{~mm}$ long). The chemical analysis of the steel conducted on a direct reading spectrometer determined its chemical composition as: $1.14 \% \mathrm{C}, 0.46 \% \mathrm{Mn}, 0.16 \% \mathrm{Si}, 0.11 \%$ $\mathrm{S}$ and $0.04 \% \mathrm{P}$. Round slices cut from the steel bar were shaped as shafts $(59 \mathrm{~mm} \times 150 \mathrm{~mm})$ by subsequent machining. The thickness of samples subjected to inhomogeneous plastic deformation were approximately $30 \mathrm{~mm}$, whereas those subjected to thermal and phase transformation were approximately $15 \mathrm{~mm}$. All these samples were made free from residual stresses by annealing them in a muffle furnace as follows: heating rate, $165^{\circ} \mathrm{C} \cdot \mathrm{h}^{-1}$; soaking time, $1 \mathrm{~h}$; soaking temperature, $850{ }^{\circ} \mathrm{C}$; cooling rate, $30^{\circ} \mathrm{C} . \mathrm{h}^{-1}$ (Fig. 5 and Fig. 6).

Residual stress measurements were made on five machined samples, three samples having thermal residual stresses and two samples having thermal and phase transformation stresses. The specimens were carefully prepared and made free from scale and dirt. Clamping of the samples to the work holder was done with chuck tightening to avoid their sliding during the experiment. Residual stresses were measured in the area located at the middle, e.g., at $1 / 2$ length in all the samples. The residual stresses were measured on the X-ray diffractometer using the strain flex X-ray analyzer. 


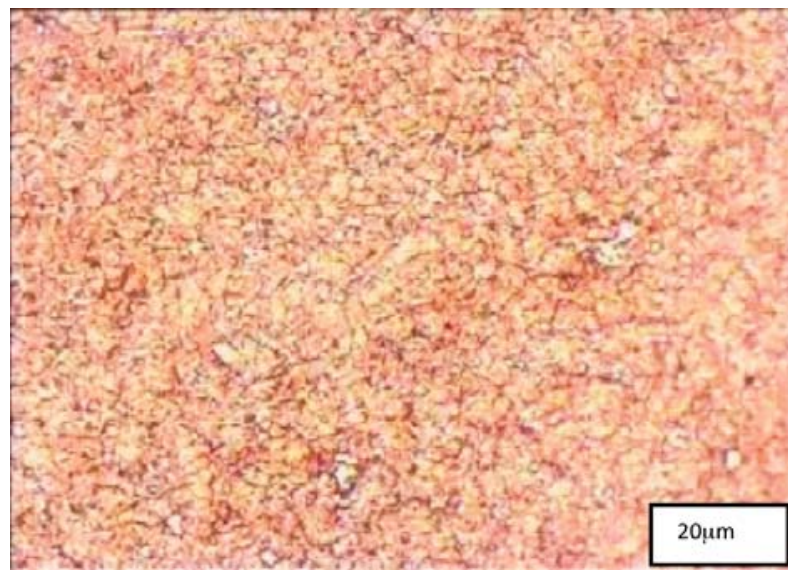

Fig. 5 Microstructure result of specimen, etching Murakami

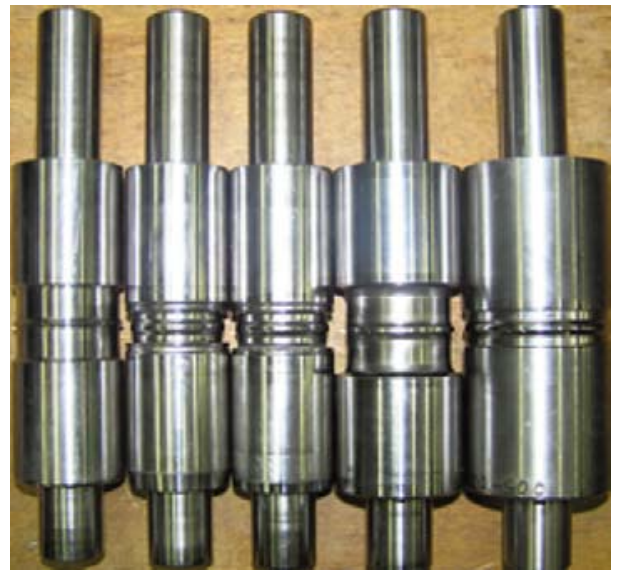

Fig. 6 Thorns on rolling bearing rings
From the theory of elasticity the relationship between residual stress $(\sigma)$ and strain $(\varepsilon)$ on the specimen surface under plane stress is given by the Bragg equation, $\lambda=2 d \sin \theta$, relating incident X-ray wavelength $(\lambda)$, lattice interplanar spacing $(d)$ and diffraction angle $(\theta)$.

Determination of the magnitude and direction of the maximum residual stress created after machining is posssible to measure by $\mathrm{X}$-ray diffractometry. The direction of maximum residual stress, that is, the most tensile or least compressive, is assumed to occur in the cutting or grinding direction during most machining operations. This is frequently the case, but the maximum stress often occurs at significant angles to the cutting direction. Furthermore, the residual stress distributions produced by many cutting operations, such as turning, may be highly eccentric, producing a highly tensile maximum stress and a highly compressive minimum stress [8].

The residual stress field at a point, assuming a condition of plane stress, can be described by the minimum and maximum normal principal residual stresses, the maximum shear stress, and the orientation of the maximum stress relative to some reference direction. The minimum stress is always perpendicular to the maximum. The maximum and minimum normal residual stresses, shown as $\sigma_{1}$ and $\sigma_{2}$ in Fig. 2, and their orientation relative to a reference direction can be calculated along with the maximum shear stress using Mohr's circle for stress if the stress $\sigma_{\varphi}$ is determined for three different values of $\varphi[9]$.

To investigate the minimum and maximum normal residual stresses and their orientation produced by turning of samples, X-ray diffraction residual stress measurements were performed in the longitudinal, $45^{\circ}$, and circumferential directions at the surface and at subsurface layers to a nominal depth of $0.1 \mathrm{~mm}$, exposing the subsurface depths by electropolishing complete cylindrical shells around

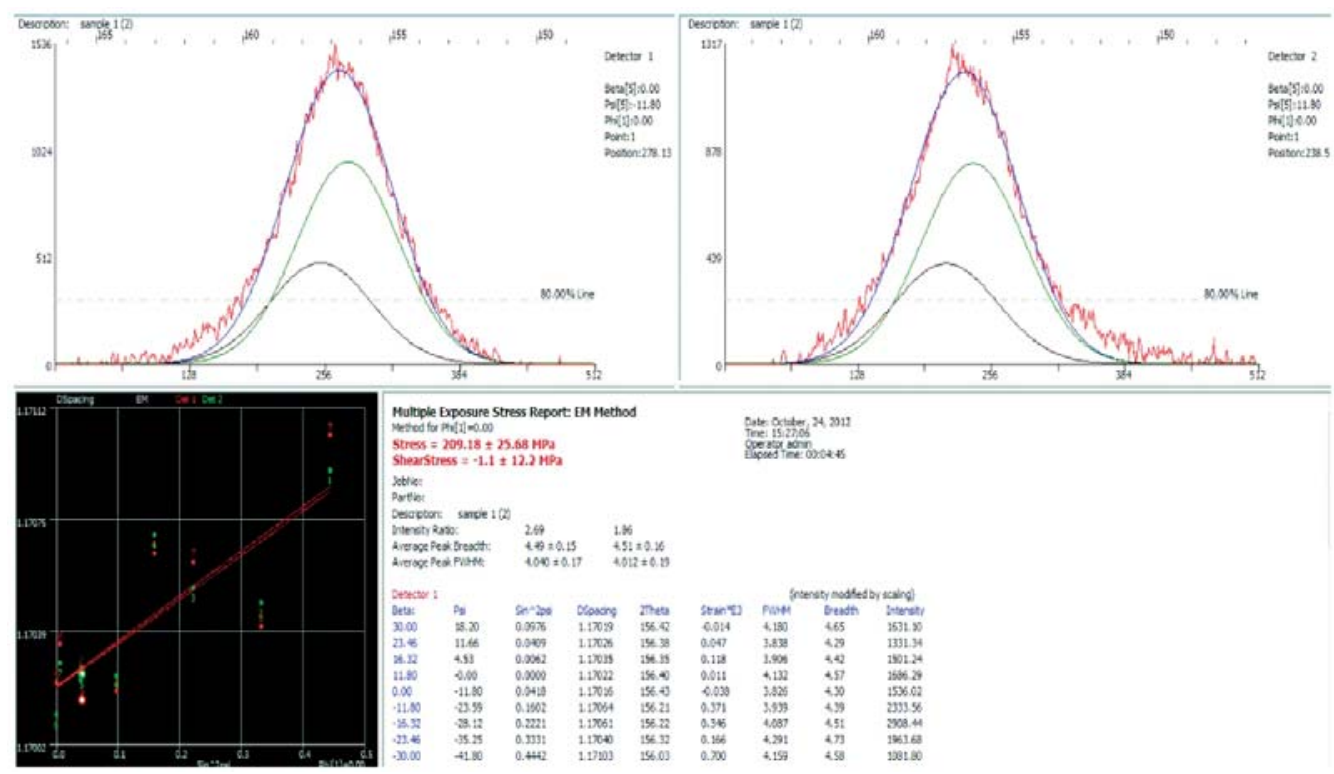

Fig. 7 Residual stress analysis of calculations with WINXRD 2.0 
the cylinder. The cylinder was nominally $59 \mathrm{~mm}$ in diameter and uniformly turned along a length of several inches. The irradiated area was limited to a nominal height of $1 \mathrm{~mm}$ around the circumference by $2.5 \mathrm{~mm}$ along the length [10]. Measurements were conducted using a $\mathrm{Cr} \mathrm{K} \alpha(420)$ two-angle technique, separating the $\mathrm{K} a_{1}$ peak from the doublet using a Cauchy peak profile (Fig. 7).

The measurements performed independently in the three directions were combined using Mohr's circle for stress at each depth to calculate the minimum and maximum normal residual stresses and their orientation defined by the angle $\varphi$, which was taken to be a positive angle counterclockwise from the longitudinal axis of the cylinder. Figure 8 illustrates the results, showing the maximum and minimum principal residual stress profiles and their orientation relative to the longitudinal direction. The maximum stresses are tensile at the surface, in excess of $140 \mathrm{MPa}$, dropping rapidly into compression at a nominal depth of $0.005 \mathrm{~mm}$. The maximum stress returns into tension at depths exceeding $0.025 \mathrm{~mm}$ and remains in slight tension to the maximum depth of $0.1 \mathrm{~mm}$ examined. The minimum residual stress is in compression in excess of $-480 \mathrm{MPa}$ at the turned surface and diminishes rapidly in magnitude with depth to less than $-138 \mathrm{MPa}$ at a depth of $0.013 \mathrm{~mm}$. The minimum stress remains slightly compressive and crosses into tension only at the maximum depth examined. The orientation of the maximum stresses is almost exactly in the circumferential direction $\left(90^{\circ}\right.$ from the longitudinal) for the first two depths examined. For depths of $0.013 \mathrm{~mm}$ to the maximum depth of $0.1 \mathrm{~mm}$, the maximum stress is within approximately $10^{\circ}$ of the longitudinal direction.

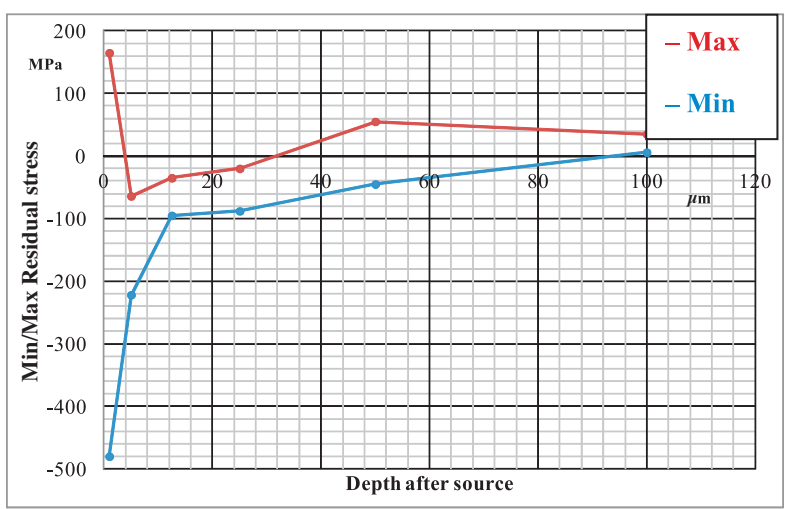

Fig. 8 Minimum and maximum principal residual stress profiles and their orientation relative to the longitudinal direction in a turned sample

\section{Experimental Results and Discussion}

To measure the residual stresses, we began by placing the parts on a pad measuring system. The detector arm focused on the unit area measured (Fig. 9). Stresses were measured in the axial and radial direction components. After preparing the parts the X-ray apparatus began to emit and X-rayed the previously selected point in the part. The device measured the stress to a depth of $12 \mu \mathrm{m}$ in the range of angles around $123^{\circ}-171^{\circ}[11]$. Then the computer formulated the results as graphs, which calculated the residual values for shear and residual stresses, which were then processed into tables.

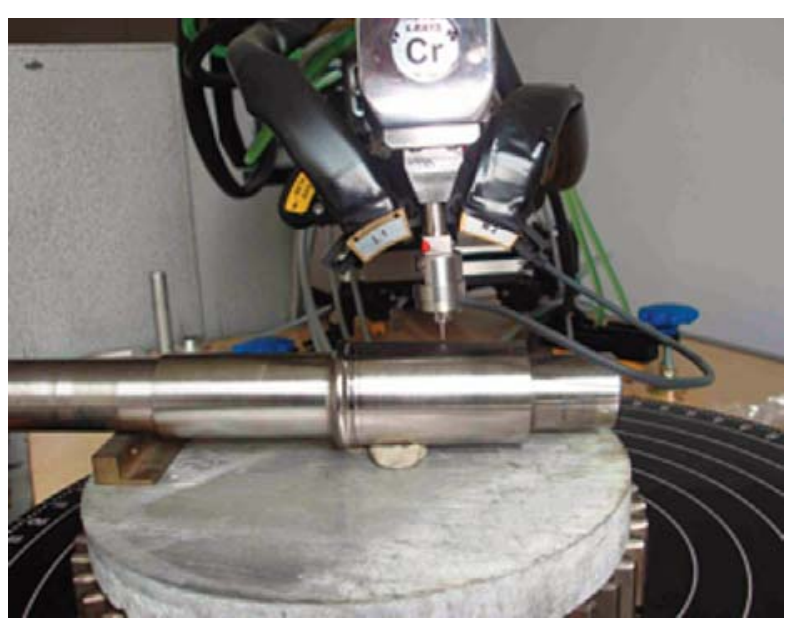

Fig. 9 Focusing the machine on the component

Turning Roughing Operation when using a cutting speed of $v_{c}=100 \mathrm{~m} \cdot \mathrm{min}^{-1}$ a measurement was made of the axially compressed nature of the residual stress, whose value hovered around $-360 \mathrm{MPa}$, and the radial residual stress in the application of the same cutting speed showed a value of $-175 \mathrm{MPa}$ pressure of the same character. With increasing cutting speed there was a reduction of the residual stresses in the axial and radial directions. With a value of $v_{c}=150 \mathrm{~m} \cdot \mathrm{min}^{-1}$ the tension in the axial direction compared with $v_{c}=100 \mathrm{~m} \cdot \mathrm{min}^{-1}$ reduced by $40 \%$, while in the axial direction it decreased by $30 \%$. When using $v_{c}=200 \mathrm{~m} \cdot \mathrm{min}^{-1}$ the tension in the axial direction when applied to the values of $v_{c}=$ $=100 \mathrm{~m} \cdot \mathrm{min}^{-1}$ decreased by $50 \%$, while in the radial direction there was a change to tensile stresses of pressure and a change of $250 \%$ (Fig. 10).

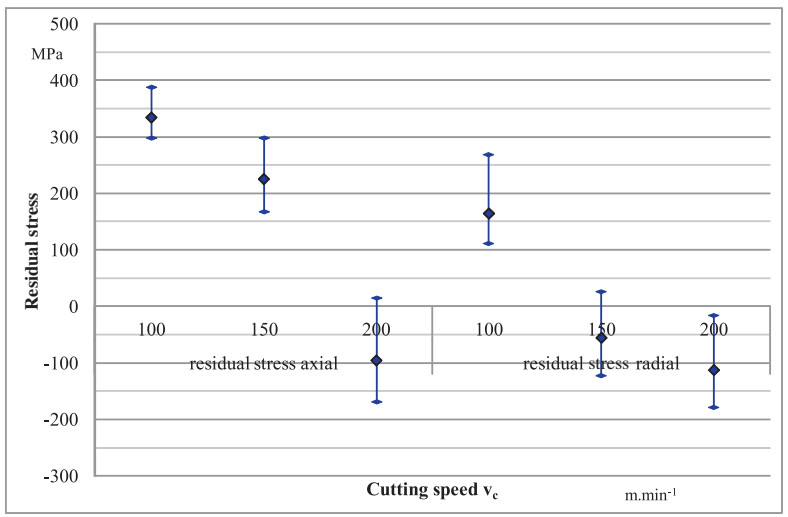

Fig. 10 Graph of residual stresses in turning roughing 
The turning operation was complete when the residual stress nature of the pressure had been finished in all cases. When using a cutting speed of $v_{c}=100 \mathrm{~m} \cdot \mathrm{min}^{-1}$ the value of the measured axial tension corresponded to a voltage of $-850 \mathrm{MPa}$, whereas the radial residual stress when applying the same cutting speed showed a value of $-580 \mathrm{MPa}$. With a cutting speed of $v_{c}=150 \mathrm{~m} \cdot \mathrm{min}^{-1}$ the tension in the axial direction increased by $2 \%$ compared with $v_{c}=$ $100 \mathrm{~m} \cdot \mathrm{min}^{-1}$ whereas in the radial direction the tension increased by $20 \%$. When using $v_{c}=200 \mathrm{~m} \cdot \mathrm{min}^{-1}$ the tension in the axial direction when applied to the values of $v_{c}=100 \mathrm{~m} \cdot \mathrm{min}^{-1}$ increased

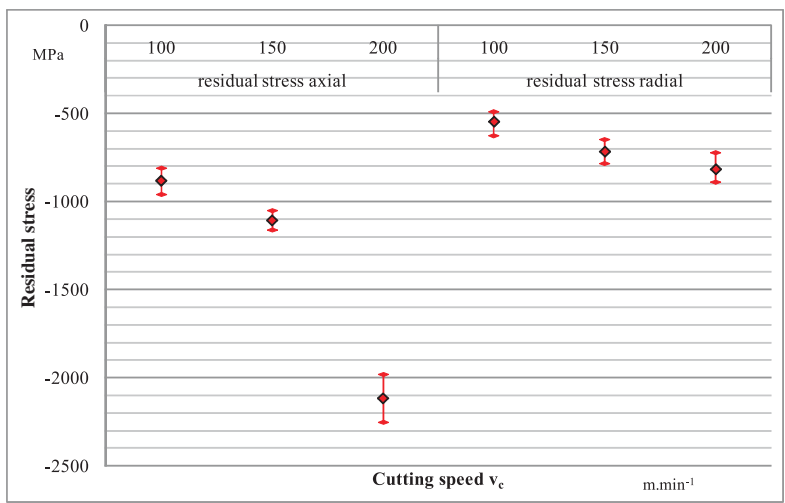

Fig. 11 Graph of residual stresses in turning finishing

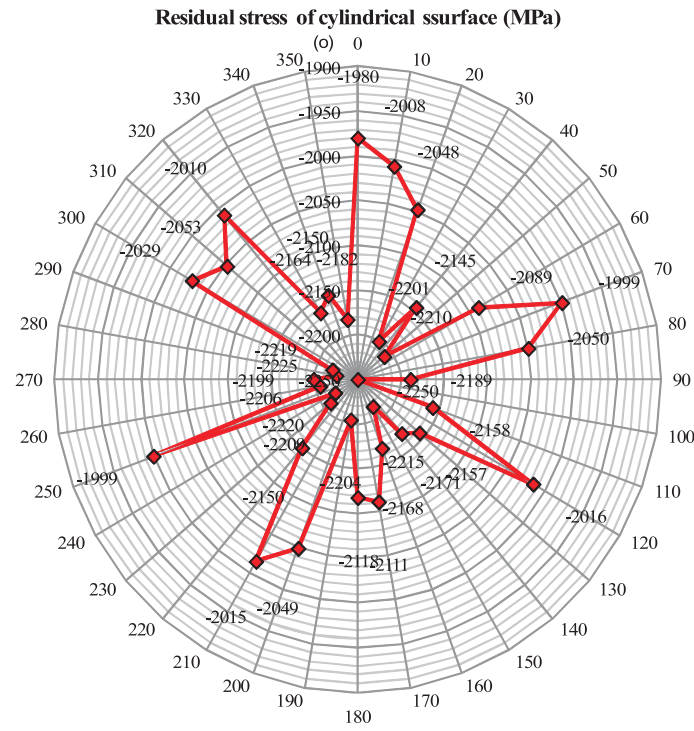

Fig. 12 Graph of residual stresses in polar coordinates when turning finishing with cutting speed $v_{c}=200 \mathrm{~m} \cdot \mathrm{min}^{-1}$ by $250 \%$, whereas in the radial direction of the residual stress it increased by $25 \%$ (Fig. 11).

That component is used as rolling tool that operates its surface on material to a ductile strength. In places where there is tensile residual stress, there is a negative effect on the functional area components. They have a great impact on the spread of cracks in components. Compressive stress occurs because the distances between the atoms themselves are very small, so they tend to associate and act against cracks in the workpiece. The compressive stress should not reach high values, for example, $2 \mathrm{GPa}$ when in the surface can give rise to crevices and cracks. The optimal value of the residual stress varies in the range of 500-700 MPa. Residual stresses in a given case should not exceed $1000 \mathrm{MP}$, and the most extreme value for steel is $2550 \mathrm{MPa}$. In this experiment, the residual stresses in some places were evaluated as $2 \mathrm{GPa}$. Such a large residual stress was caused by previous use of the rolling thorn, which created great forces that had a large impact on the results of the measurements of residual stresses in this experiment (Fig. 12).

The results appear to indicate that stresses within approximately $0.013 \mathrm{~mm}$ of the sample surface are dominated by machining, which resulted in a maximum stress direction essentially parallel to the cutting action. At greater depths, the stress distribution may be governed not so much by the machining as by stresses that may have been present due to forging or heat treatment.

\section{Conclusion}

Distributions of residual stresses on the surfaces and along the depth of the machining steel samples have been presented. The stress distribution for the sample with the cylinder sample is characterised by compressive stresses on the surface and by tensile stresses in the subsurface. Residual stress distributions for samples with a circular surface are more complicated.

Conclusions about reversing of compressive residual stress on the surface of the sample to compressive and tensile stress in the depth made by analysis of equilibrium equations have been confirmed experimentally.

This work is related to the project with the University of Zilina, 2009/2.2/04-SORO OPVaV number (26220220101), and named "Intelligent system for non-destructive evaluation technologies for functional properties of components of X-ray diffractometry". The main objective is to transform the new non-destructive technologies for knowledge transfer to industry practice in the evaluation of functional properties of the surface and subsurface layers of non-destructive techniques.

\section{References}

[1] GRIFFITHS, C. A.: Closing the Loop on Product Integrity on Bearings with Engineered Source Approval, Split Ballbearing Features, Company Newsletter, July 1989 
[2] TIITTO, K. et al.: Testing Shot Peening Stresses in the Field, The Shot Peener, vol. 4, 1991, ISSN: 1069-2010

[3] PREVEY, P. S.: X-ray Diffraction Residual Stress Techniques, Metals Handbook 10, Metals Park : American Society for Metals, 1986, 380-392. ISSN: 0096-7416

[4] PREVEY, S., WOJTAS, A.S.: Future of Residual Stress Testing in Shot Peened Parts During Processing, SURFAIR IX - 9th Intern. Conference on Surface Treatments in Aeronautic and Aerospace Industry, Cannes, 1992, ISBN 0375502777

[5] TIITTO, S.: On the Influence of Microstructure on Magnetization Transitions in Steel, Acta Polytechnica Scandinavica, Applied physics series No. 119, Helsinki, 1977

[6] JACOB, P., MARRONE, S.: Non-destructive Evaluation of Residual Stress Depth-profiles by Barkhausen Noise Analysis and their Validation by XRD Method Combined with Electrochemical (destructive) Surface Removal, $4^{\text {th }}$ Inter. Conference on Barkhausen Noise and Micromagnetic Testing, 2003, Brescia - Italy, ISBN: 951-98400-4-4

[7] SAPIETOVA, A., SAGA, M., NOVAK, P.: Multi-software Platform for Solving of Multibody Systems Synthesis, Comunications Scientific Letters of the University of Zilina, vol. 14, No. 3, 43-48, 2012, ISSN 1335-4205

[8] HANDRIK, M., JAKUBOVICOVA, L., KOPAS, P., SAGA, M.: Analysis of Microplastic Areas Near Graphite Particles of Nodular Cast Iron Loading bellow Yield Stress. Metalurgija (Metallurgy), vol. 49, No. 2, 2010, 263-267, ISSN 0543-5846

[9] ZMINDAK, M., RIECKY, D.: Meshless Modelling of Laminate Mindlin Plates under Dynamic Loads Synthesis, Comunications - Scientific Letters of the University of Zilina, vol. 14, No. 3, 2012, 24-31, ISSN 1335-4205

[10] NESLUSAN, M., CZAN, A.: Uzurpel Analysis of the Heat Distribution when Grinding of a VT 9 Titanium Alloy and its Relation to Residual Tresses, Strojniski vestnik - J. of Mechanical Engineering, vol. 48, No. 10, 557-564, 2002, ISSN 0039-2480

[11] CEP, R., OCENASOVA, L., NOVAKOVA, J., PETRKOVSKA, L., CZAN, A., STANCEKOVA, D.: Interrupted Machining Tests of Ceramic Cutting Tools, TMT 2009 Proc., October 2009, Hammamet, vol. 13, No. 1, 733-736, ISSN 1840-4944. 